\title{
Genetic mutations and mechanisms in dilated cardiomyopathy
}

\author{
Elizabeth M. McNally, Jessica R. Golbus, and Megan J. Puckelwartz
}

Department of Human Genetics, University of Chicago, Chicago, Illinois, USA.

\begin{abstract}
Genetic mutations account for a significant percentage of cardiomyopathies, which are a leading cause of congestive heart failure. In hypertrophic cardiomyopathy (HCM), cardiac output is limited by the thickened myocardium through impaired filling and outflow. Mutations in the genes encoding the thick filament components myosin heavy chain and myosin binding protein C (MYH7 and MYBPC3) together explain $75 \%$ of inherited HCMs, leading to the observation that HCM is a disease of the sarcomere. Many mutations are "private" or rare variants, often unique to families. In contrast, dilated cardiomyopathy (DCM) is far more genetically heterogeneous, with mutations in genes encoding cytoskeletal, nucleoskeletal, mitochondrial, and calcium-handling proteins. DCM is characterized by enlarged ventricular dimensions and impaired systolic and diastolic function. Private mutations account for most DCMs, with few hotspots or recurring mutations. More than 50 single genes are linked to inherited DCM, including many genes that also link to HCM. Relatively few clinical clues guide the diagnosis of inherited DCM, but emerging evidence supports the use of genetic testing to identify those patients at risk for faster disease progression, congestive heart failure, and arrhythmia.
\end{abstract}

\section{Cardiomyopathy: diagnosis and prevalence}

Cardiomyopathy is defined by a pathologically abnormal myocardium. There are four major classifications of cardiomyopathy: dilated (DCM), hypertrophic (HCM), restrictive (RCM), and arrhythmogenic RV (ARVC). Cardiomyopathy is most commonly diagnosed through in vivo imaging, with either echocardiography or, increasingly, cardiac MRI. DCM refers to enlargement of the heart, which often affects all four chambers, especially late in the disease. Most commonly, DCM is associated with reduced LV function or systolic function, although early in the disease the LV may be dilated, with only minimally reduced function. In contrast, HCM is characterized by increased LV wall thickness, often targeting the septum that separates the LV from the RV (Figure 1). RCM is the most elusive, in part because the heart may appear morphologically close to normal, with only minor increased wall thickness or modestly decreased LV ejection fraction. The infiltrative process underlying RCM is often not readily detectable in vivo with even the most sensitive imaging technique. RCM is characterized by impaired filling of the heart, known as diastolic dysfunction, which reduces cardiac output. ARVC is characterized by reduced function and thinning of the RV with a fibrofatty infiltration that can be seen on MRI.

Myocardial ischemia remains a common cause of DCM, accounting for approximately half of DCM. Toxic, metabolic, and immunologic causes have each been linked to DCM, as well as hypertension and valvular disease. Genetic cardiomyopathy, which runs in families, is now more commonly diagnosed, largely because surveillance for and awareness of the condition have improved. It is also now increasingly understood that more than one etiology may contribute to DCM within a given individual. Unlike cardiomyopathy that results from some of the other causes, genetic cardiomyopathy is a progressive disease. The time course is highly dependent on the age of presentation, which relates to

Conflict of interest: An immediate family member of E.M. McNally receives patent royalties related to massively parallel sequencing.

Citation for this article: J Clin Invest. 2013;123(1):19-26. doi:10.1172/JCI62862. the specific genetic mutation as well as the presence of disease modifiers. Because it is now commonplace to screen relatives of individuals affected with cardiomyopathy, many individuals are diagnosed at much earlier stages of disease. This detection strategy is expected to change both the prevalence and treatment strategies for cardiomyopathy. Previous estimates of DCM prevalence were 1 in 2,500-3,000 $(1,2)$. However, these data reflect older diagnostic modalities and therefore likely significantly underestimate the disease prevalence. For HCM, previous estimates were similar to DCM, but newer modalities and heightened diagnostic awareness now estimate the prevalence of HCM to be 1 in 500 (3). The prevalence of DCM increases with age, and DCM is rare (1-2 in 100,000) in the pediatric population (4). DCM is also more common among males, and prevalence varies with ethnicity. Nonischemic DCM, defined as DCM not from myocardial ischemia or infarct, is familial in $25 \%-50 \%$ of cases, with estimates that vary based how family members were screened (5).

\section{Genetic mutations in DCM}

Familial DCM refers to DCM that is inherited as a single gene disorder in a Mendelian pattern. The primary mode of inheritance for familial DCM is autosomal dominant, with reduced penetrance and variability expressivity. Mitochondrial mutations also contribute to DCM with the expected matrilineal inheritance. Autosomal-recessive mutations are a less common cause of familial DCM, although X-linked recessive inheritance is associated with several genes such as dystrophin, tafazzin, and emerin. At least 50 single genes have been identified as linked to familial DCM, and the majority of these elicit disease as dominant mutations. With few exceptions, it is not possible to determine which gene is responsible for DCM on clinical grounds. That is, mutations in many single genes phenocopy DCM. Because mutations in many individual single genes lead to DCM, genetic testing commonly employs multi-gene panels, in which more than 50 genes can be tested simultaneously. With the use of multi-gene panels for clinical genetic testing, more than a single pathogenic variant can be identified within an individual, thus explaining some features of 

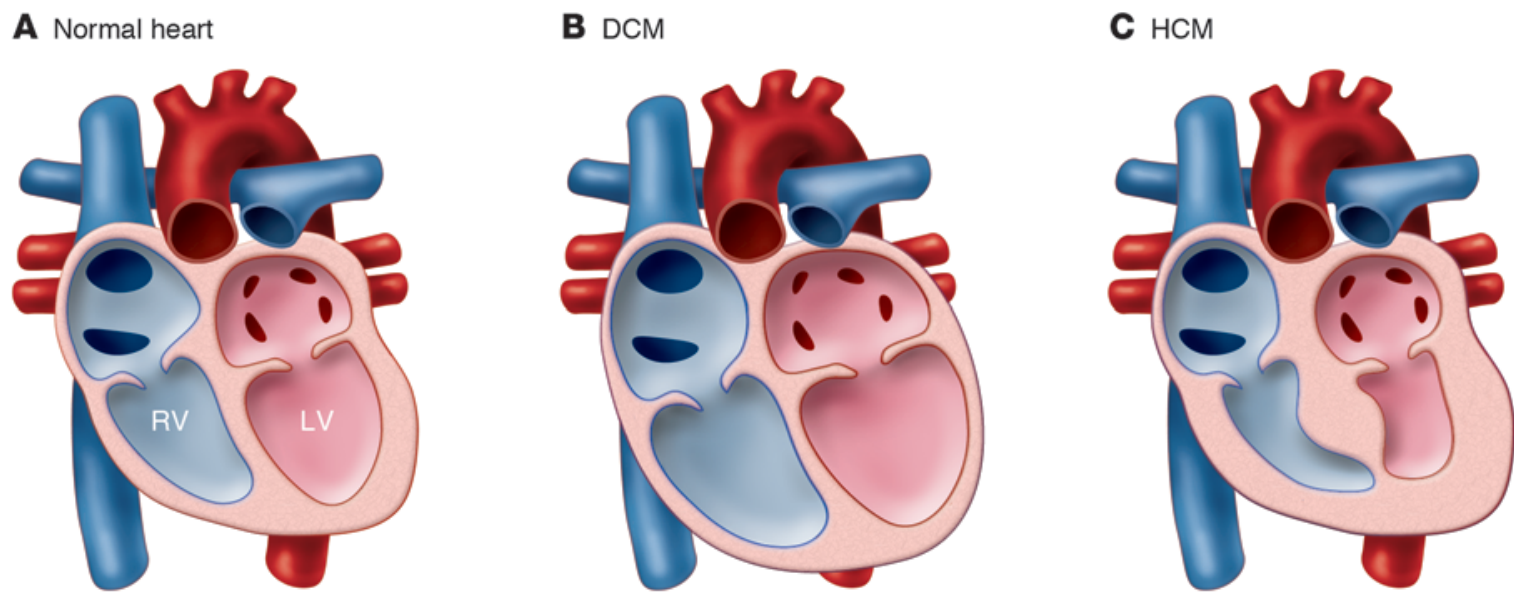

\section{Figure 1}

Morphological changes to the heart in cardiomyopathy. (A) Normal heart. (B) In DCM, the heart enlarges with increased diameter and reduced function. (C) In HCM, the myocardium - especially in the LV - becomes thickened, leading to impaired filling and emptying.

early onset in more severe forms of disease $(6,7)$. Some of the genes linked to DCM encode proteins of the sarcomere, costamere, $\mathrm{Z}$ band, and nuclear membrane, while others have functions distinct from these broad cell biological classifications (Table 1).

Sarcomere DCM genes. The sarcomere is the unit of contraction for striated muscle (Figure 2). Thin filaments emanate from the $\mathrm{Z}$ band with filamentous actin (gene name ACTC1) forming the core and tropomyosin (encoded by TPM1) and the troponin subunits (encoded by TNNT2, TNNC1, TNNI3) providing calcium regulation of the actomyosin interface. The thick filament core is formed by $\beta$-myosin heavy chain ( $\beta$-MHC) (encoded by MYH7), with myosin binding protein $\mathrm{C}$ (MYBPC3) highly concentrated in the $\mathrm{M}$ band region. The giant protein titin (encoded by TTN) spans the length of half the sarcomere, where it acts as a stretch sensor, transmitting signals from its anchor at the $\mathrm{Z}$ band to its carboxyterminal kinase domain. Dominant mutations in the genes encoding these sarcomeric proteins cause DCM. Many sarcomeric gene mutations are nonsynonymous SNPs, small insertion/deletions that may be in-frame or, alternatively, protein-truncating mutations. An estimated $35 \%-40 \%$ of genetic DCMs may result from sarcomere gene mutations, with the majority attributed to protein-truncating mutations in TTN (8). Notably, arrhythmia risk may not correlate with morphological abnormality (9), and additional markers to predict arrhythmia risk are needed.

The mechanism of action for some sarcomeric DCM mutations is dominant negative. A gain of function by the protein produced from the mutant allele interferes with sarcomere formation and function. The experimental evidence to support expression of the mutant allele is limited by the availability of human hearts and often relies on demonstration of variant pathogenicity within an experimental model. The deletion of lysine at position 210 (del210K) in TNNT2 has been linked to DCM (10). Although initially believed to be a highly potent mutation with early onset and lethal disease, subsequent reports revealed the TNNT2 del210K mutation to be associated with a marked phenotypic expression range with both young and older affected individuals (11). The TNNT2 del210K mutation was engineered as a knockin allele in the mouse, where it produced DCM and reduced survival
(12). Skinned cardiac muscle fibers from heterozygous del210K mice showed decreased calcium sensitivity for force generation (12). Homozygous TNNT2 del210K mice showed an even more enhanced rightward shift of decreased calcium sensitivity, markedly enlarged hearts, and further reduced survival compared with heterozygous TNNT2 del210K mice. These findings suggest that TNNT2 del210K disrupts intermolecular interactions within the sarcomere, most likely with tropomyosin.

One recent study of a TNNC1 DCM mutation used mass spectrometry to document equimolar expression of the G159D allele and the normal TNNC1 allele in an explanted human DCM heart (13). The calcium sensitivity of force generation was increased with this mutation, in opposition to what occurs with the TNNT2 mutation described above. Tropomyosin is a thin filament protein that is also mutated in DCM. For example, the TPM1 D230N mutation was described in two large, unrelated families with DCM (14). As with TNNT2 del210K, the DCM clinical phenotype with TPM1 D230N markedly varied. Some children presented with cardiomyopathy, while other family members with the identical gene mutation remained largely asymptomatic, with mildly increased LV diameter and mildly reduced systolic function until later life. The D230N TMP1 DCM mutation was modeled in vitro, where a decreased calcium sensitivity of actin-activated myosin ATPase activity was seen (14). This result is in contrast to the increased calcium sensitivity seen with an HCM-inducing TPM1 D175N mutation. Sarcomere mutations have been well studied using enzymatic assays of myosin function (e.g., assays of actin-activated myosin ATPase activity) and more complex biophysical assays of actin's sliding filament velocity, which can be further interrogated to monitor in vitro force production. Isolated muscles, usually the papillary muscle, are treated with detergents to strip away membrane components and leave intact, skinned fibers. These reductionist approaches (in vitro motility or isolation of fibers) simplify the sarcomere, often leading to useful results. However, the results should be cautiously interpreted, especially where there are inconsistencies, because these approaches do not replicate the full complexity of sarcomere function within a failing heart (for a more full discussion, see ref. 15). 
Table 1

Cardiomyopathy genes

\section{Gene}

ABCC9

ACTC1

ACTN2

ANKRD1

BAG3

CASQ2

CAV3

COX15

CRYAB

CSRP3

CTF1

DES

DMD

DNAJC19

DSC2

DSG2

$D S P$

DTNA

EMD

EYA4

FHL2

FKTN

FOXD4

GLA

JUP

LAMA4

LAMP2

$\angle D B 3$

LMNA

MYBPC3

MYHG

MYH7

MYL2

MYL3

MYLK2

MYOZ2

NEXN

PKP2

PLN

PRKAG2

PSEN1

PSEN2

RBM20

RYR2

SCN5A

SDHA

$S G C D$

SYNE1

SYNE2

TAZ

TCAP

TMEM43

TMPO

TNNC1

TNNI3

TNNT2

TPM1

TTN

TTR

$V C L$
Gene name

ATP-binding cassette, sub-family C, member 9 Actin, $\alpha$, cardiac muscle 1 Actinin, $\alpha 2$

Ankyrin repeat domain 1 (cardiac muscle)

BCL2-associated athanogene 3

Calsequestrin 2 (cardiac muscle)

$$
\text { Caveolin } 3
$$

C0X15 homolog, cytochrome $\mathrm{c}$ oxidase assembly protein Crystallin $\alpha \mathrm{B}$

Cysteine and glycine-rich protein 3

Cardiotrophin 1

Desmin

Dystrophin

DnaJ (Hsp40) homolog, subfamily C, member 19

Desmocollin 2

Desmoglein 2

Desmoplakin

Dystrobrevin, $\alpha$ Emerin

Eyes absent homolog 4

Four and a half LIM domains 2

Fukutin

Forkhead box D4

Galactosidase, $\alpha$

Junction plakoglobin

Laminin, $\alpha 4$

Lysosomal-associated membrane protein 2

LIM domain binding 3 Lamin A/C

Myosin binding protein $\mathrm{C}$, cardiac

Myosin, heavy chain 6 , cardiac muscle, $\alpha$

Myosin, heavy chain 7 , cardiac muscle, $\alpha$

Myosin, light chain 2, regulatory, cardiac, slow

Myosin, light chain 3, alkali; ventricular, skeletal, slow

Myosin light chain kinase 2

Myozenin 2

Nexilin (F actin binding protein)

Plakophilin 2

Phospholamban

Protein kinase, AMP-activated, $\gamma 2$, non-catalytic subunit

Presenilin 1

Presenilin 2

RNA binding motif protein 20

Ryanodine receptor 2 (cardiac)

Sodium channel, voltage-gated, type V, $\alpha$ subunit

Succinate dehydrogenase complex, subunit A, flavoprotein Sarcoglycan, $\delta$

Spectrin repeat containing, nuclear envelope 1

Spectrin repeat containing, nuclear envelope 2 Tafazzin

Titin-cap (telethonin)

Transmembrane protein 43

Thymopoietin

Troponin C type 1 (slow)

Troponin I type 3 (cardiac)

Troponin T type 2 (cardiac)

Tropomyosin $1(\alpha)$ Titin

Transthyretin

Vinculin
Cardiomyopathy subtype(s)

DCM

DCM, HCM, LVNC

$\mathrm{DCM}, \mathrm{HCM}$

DCM, HCM

DCM

LVNC, CPVT

$\mathrm{HCM}$

$\mathrm{HCM}$

$\mathrm{HCM}$

DCM, HCM

DCM

DCM

DCM

DCM

DCM, ARVC

ARVC

DCM, ARVC

LVNC

DCM

DCM

DCM

DCM

DCM

HCM

ARVC

DCM

DCM, HCM

DCM, LVNC

DCM, LVNC

DCM, HCM, LVNC

DCM, HCM

DCM, HCM, LVNC

$\mathrm{HCM}$

$\mathrm{HCM}$

$\mathrm{HCM}$

$\mathrm{HCM}$

DCM, HCM

ARVC

DCM, HCM

$\mathrm{HCM}$

DCM

DCM

DCM

CPVT

DCM

DCM

DCM

DCM

DCM

DCM, LVNC

DCM

ARVC

DCM

DCM, HCM

DCM, HCM

DCM, HCM, LVNC

DCM, HCM

DCM, HCM, ARVC

$\mathrm{HCM}$

DCM, HCM

CPVT, catecholeminergic polymorphic ventricular tachycardia; LVNC, LV noncompaction. 
A

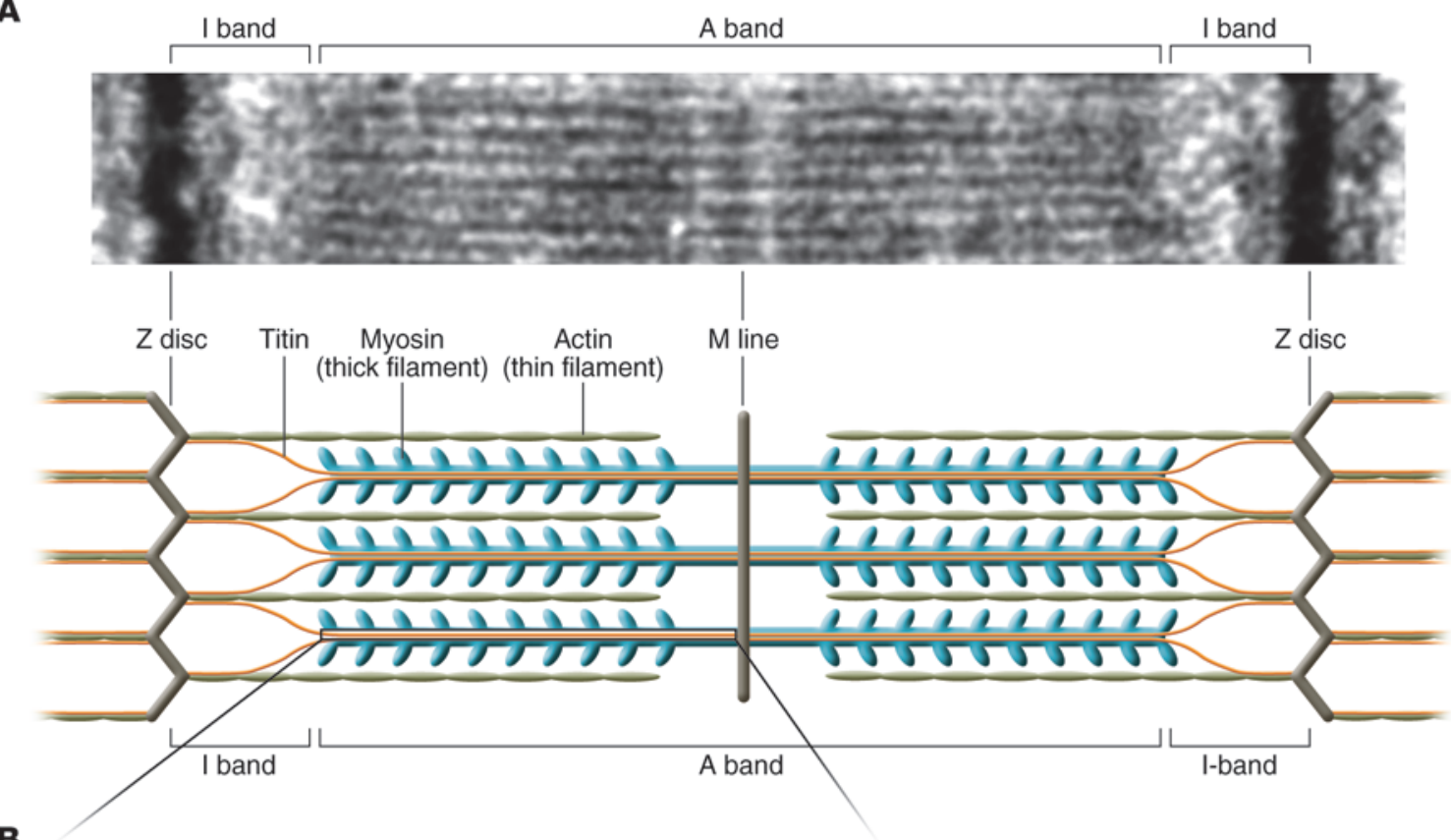

B

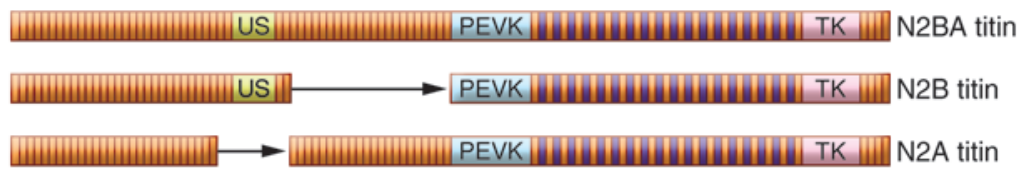

】lg domain \Fn domain

\section{Figure 2}

The sarcomere is a target for cardiomyopathy mutations. (A) The sarcomere from $Z$ disc to $Z$ disc. Electron micrograph of a sarcomere from a human heart. The TTN gene encoding the giant protein titin is mutated in DCM. Titin's amino terminus anchors in the $Z$ band, and its carboxy terminus ends in the $\mathrm{M}$ band. The titin kinase (TK) domain is found at the carboxy terminus and, when mutated, results in impaired stretch sensing and signaling. Titin interacts with both the thin and thick filaments. The thick-filament proteins (grey) are encoded by MYH7 and MYBPC3, two genes commonly linked to HCM. (B) Isoforms of titin. Alternative splicing in the region of titin that encodes the I band gives rise to isoforms with varying spring properties. The N2B isoform is found exclusively in cardiac muscle and the N2A isoform in skeletal muscle. The N2BA isoform is also found in cardiac muscle and contains features found in both N2B and N2A titin. N2BA titin has a longer extensible I band region than N2B titin, making it more compliant. US, unique sequence; Fn, fibronectin domains; PEVK, repeating units of amino acids (proline, glutamic acid, valine, and lysine).

DCM mutations also occur in the sarcomere's thick filament (16). MYH7 and MYBPC3 mutations are the most common causes of HCM, and HCM may progress to DCM. However, there are thick filament mutations that have no HCM phase. Two MYH7 mutations, S532P and F764L, each linked to familial DCM, were independently modeled in mice by modifying the endogenous $\alpha$-MHC (encoded by MYH6), which is the major $\mathrm{MHC}$ of the small rodent heart. Each of these mutations maps within the $\mathrm{S} 1$ head of myosin that harbors the actin-binding, ATPase, and force-producing domains. The S532P mutation occurs within the actin-binding domain, and the F764L mutation occurs within the converter domain. Actin-activated ATPase is reduced, as is in vitro sliding motility of actin. Force production is not decreased, but rather a reduction in myosin step size is observed. Since ATP hydrolysis produces less displacement of myosin, this is a less energetically efficient sarcomere. Mutations in MYH7 have also been described in patients with LV noncompaction cardiomyopathy (LVNC), a pathological variant with deep trabecular invaginations in the LV. Truncating and point mutations in MYH7 have been identified in LVNC families (17). In addition, TPM1 and MYBPC 3 mutations have also been found in LVNC patients $(17,18)$.

Most recently, mutations in the giant protein titin were estimated to be responsible for approximately $25 \%$ of DCM (8). The titin protein includes more than 35,000 amino acids, containing many repeating fibronectin-like and Ig-like domains. Titin is considered a molecular ruler that regulates and transmits information about sarcomere length to the sarcomere and the cardiomyocyte (19). Consistent with this role, the carboxy terminus titin kinase domain is stimulated by activity and stretch (20). The TTN gene at chromosome 2 was previously linked to familial DCM (21), and those findings have been reinforced by studies in animal models (22). To evaluate N2BA, one of the numerous isoforms encoded by the TTN gene, an exon-capture strategy was employed. The N2BA form contains additional domains compared to the N2B form, including the Ig and PEVK domains that lend additional recoil (23). In heart failure, the ratio of N2BA to N2B is altered, which may be both a cause and effect of cardiac ventricular dilation (24). 


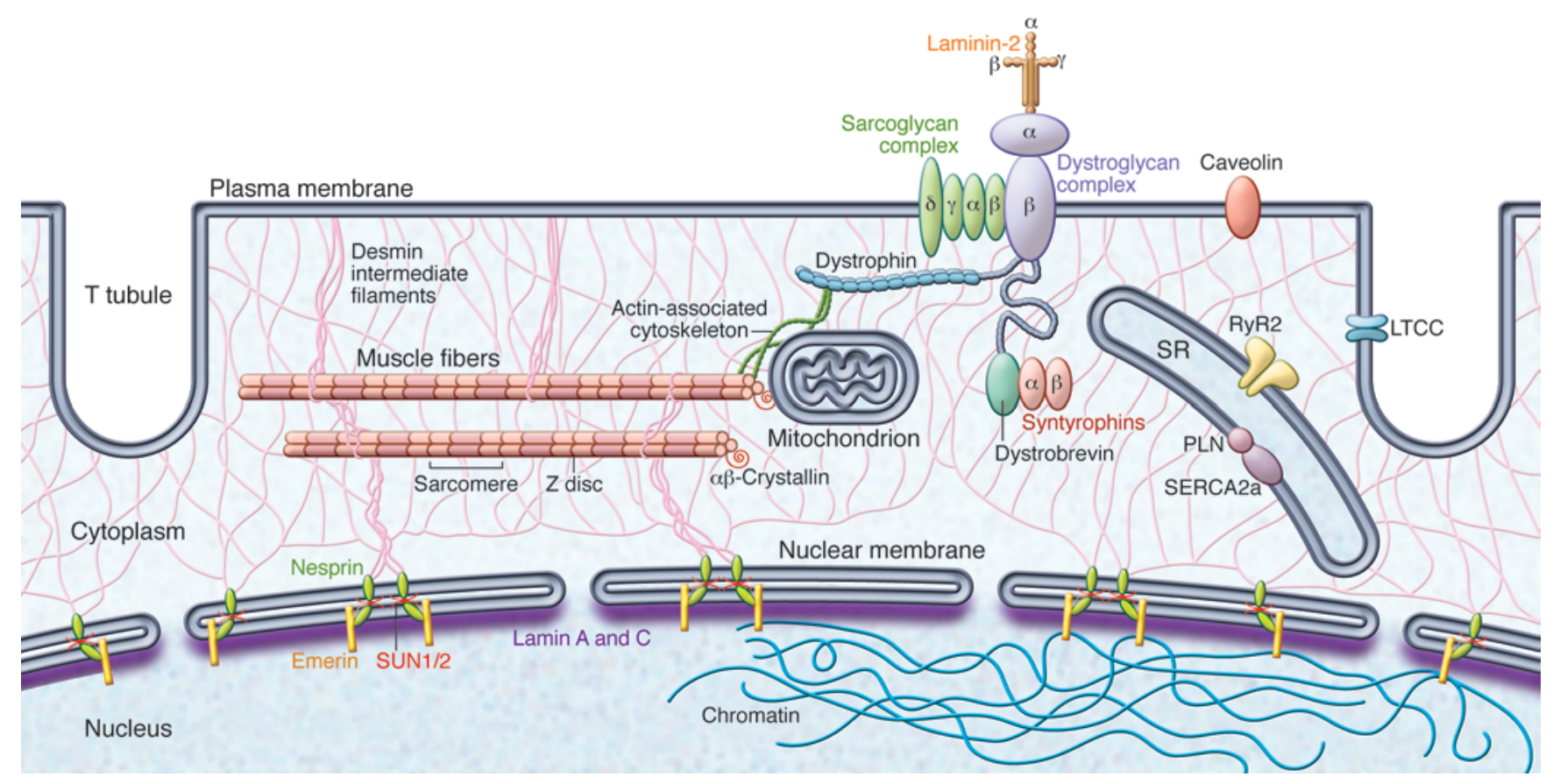

Figure 3

A view of the cardiomyocyte. The cytoplasm of the cardiomyocyte contains sarcomeres, which contain thin and thick filaments. Mutations in sarcomere genes lead to HCM and DCM. Plasma membrane-associated proteins such as dystrophin and its associated proteins, the sarcoglycans, are mutated in inherited DCM associated with skeletal muscle disease. Mutations of genes encoding nuclear membrane proteins such as lamins $\mathrm{A}$ and C, emerin, and nesprins lead to inherited DCM due to an inappropriate transcriptional response to mechanical stress. Many of these nuclear membrane genes also induce cardiac conduction system disease.

Many of these same mutations are also expected to alter skeletal muscle titin, which is encoded by the related isoform N2-A titin, but whether these variants contribute to subclinical muscle disease remains to be documented.

A dilated heart may be even more dependent on signaling through titin, and frameshifting mutations that disrupt this intramolecular signaling pathway would be expected to adversely affect the heart, as it regularly contends with repetitive and dynamic load. In the study by Herman et al., 54 of 312 DCM patients were found to have truncating TTN mutations, which the authors defined as frameshifts, nonsense, or predicted splice site mutations (8). In comparison, only 3 of $231 \mathrm{HCM}$ cases and, intriguingly, 7 of 249 control subjects had protein-disrupting TTN mutations. The protein-disrupting TTN variants were nonrandomly distributed along the length of titin, with a preference for the A band region of titin. Most TTN variants mapped in regions common to both the N2B and N2BA forms, and nearly all TTN mutations were heterozygous. A subset of subjects had documented familial disease, thus providing strong support for TTN as a dominant DCM gene. In addition to these protein-truncating TTN mutations, a large number of TTN coding variants of unknown significance were found. This type of protein-altering variation may contribute to DCM complexity, as some variants are predicted to be pathogenic. With this frequency of protein-disrupting variation, the TTN gene should be investigated in DCM, although the challenges associated with evaluating the large and repetitive TTN gene are enormous. Given this frequency, TTN mutations in combination with other forms of heart disease would be expected to enhance the severity of cardiomyopathy expression. For example,
$T T N$ variants in combination with valve defects, hypertension, or myocardial infarction would be expected to result in more significant cardiomyopathy and congestive heart failure. A larger heart, such as that dilated from regurgitant valve disease, may be more susceptible to impaired elastic recoil or a reduction in titin signaling from mutations ablating the titin kinase domain. It also follows that TTN mutations in combination with other pathogenic genetic variants would enhance the severity of DCM. It is notable that HCM subjects had a strikingly lower percentage of TTN frameshifting mutations in the Herman study, possibly supporting the idea that HCM sarcomere mutations along with TTN
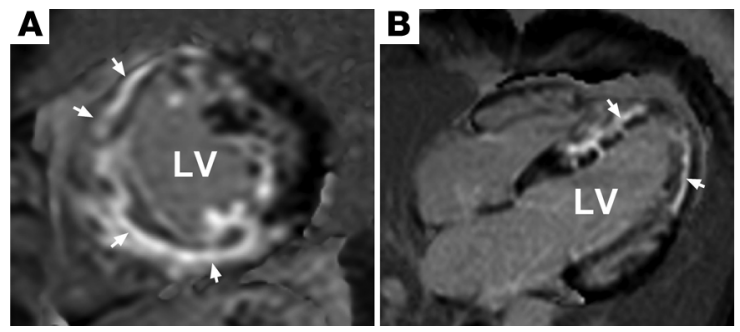

\section{Figure 4}

Fibrosis occurs commonly in DCM. Cardiac MRI of a patient with an MYH7 mutation. (A) Short-axis view sectioning through the LV shows late gadolinium enhancement, which indicates fibrosis. Late gadolinium enhancement is visualized as white regions (arrows) and is never seen in a normal heart. (B) Four-chamber view shows late gadolinium enhancement (arrows) in the same MYH7 mutant individual. 
frameshifting mutations predispose to DCM. More comprehensive genetic testing is required to explore this possibility, and these observations strongly favor the inclusion of TTN in genetic screening panels. TTN variants have also been described in ARVC (25).

$Z$ band proteins and the costamere. The $Z$ disc or $Z$ band is an electron-dense structure into which the thin filaments and titin anchor. Gene mutations that have been identified in DCM patients that affect multiple $Z$ band proteins include muscle LIM protein (MLP), cardiac ankyrin repeat protein (CARP), myopallidin, $\alpha$-actinin 2, TCAP, and nexilin (26-32). TCAP and MLP modulate the elastic properties of titin. Phosphorylation of $\mathrm{Z}$ band proteins, or even nuclear translocation of $Z$ band elements, are just two mechanisms by which $Z$ band elements signal within the cardiomyocyte.

At the plasma membrane, with a periodicity that matches $Z$ discs, is the costamere, a rib-like structure of cytoplasmic and transmembrane proteins that links the cytoskeleton to the plasma membrane and the extracellular matrix (Figure 3). Dystrophin and its associated proteins, sarcoglycans and dystroglycan, enrich at the costamere. Linked to the cytoplasm alongside talin, vinculin, and integrin, the dystrophin complex protects against contraction-induced injury in both cardiac and skeletal muscle (33). The integrity of the dystrophin complex is critical for mechanotransduction because disruption of this complex from loss-of-function mutations renders cardiomyocytes susceptible to damage elicited from contraction (34). Mutations in dystrophin and the sarcoglycan produce skeletal muscle disease and cardiomyopathy, and so heart failure in these patients is further compromised by hypoventilation from respiratory muscle weakness.

Because of the narrow window in which DCM develops in patients with Duchenne muscular dystrophy (DMD), this population has been ideal for testing whether medications for cardiomyopathy can prevent or slow progression of DCM. DCM prevention was tested by giving the angiotensin converting enzyme perindopril to boys with DMD who began treatment beginning at age 9.5 years, before the development of reduced LV function. This early treatment was associated with reduced progression of cardiomyopathy after five years and also showed a benefit in 10-year mortality (35, 36 ). The addition of $\beta$-adrenergic blockade may be expected to further slow progression of disease. Although animals have been used to test whether early treatment slows progression of DCM, the perindopril study in a human population with DMD provides strong evidence to support what was seen using animal models.

Nuclear membrane defects in genetic DCM. Lamins A and C, two alternatively spliced products of the $L M N A$ gene, are expressed in all somatic cells, but mutations in LMNA strike in a very tissuespecific manner, with the majority causing cardiomyopathy with variable skeletal muscle involvement. More than 200 different LMNA mutations are associated with inherited cardiomyopathy, primarily DCM that may be associated with conduction system disease prior to the evidence of ventricular dilation. LMNA mutations that cause DCM are primarily inherited in an autosomaldominant manner, although autosomal-recessive cases have been reported. Cardiac conduction system disease caused by LMNA mutations includes sinus atrial node disease, atrial dysrhythmias, atrioventricular heart block, and ventricular tachyarrhythmias (37). In a study of patients with familial DCM and prominent conduction block, $33 \%$ had LMNA mutations (38). A recent report found that frameshifting LMNA mutations have the highest risk for ventricular arrhythmias (39).
Lamins $\mathrm{A}$ and $\mathrm{C}$ are intermediate filament proteins that are present at the nuclear membrane and in the nucleoplasm. Lamins A and $\mathrm{C}$, along with the B-type lamins and other lamina-associated proteins, comprise the nuclear lamina, a fibrous structure that provides support to the nuclear membrane. The lamina proteins are part of the LINC complex that LInks the Nucleus to the Cytoplasm. In addition to lamins A and C, mutations in other members of the LINC complex cause cardiomyopathy, including emerin and nesprins- 1 and -2 (40-42). Stress signals in the cytoplasm are hypothesized to act on the LINC complex to affect gene expression in the nucleus. Both LMNA and emerin-null fibroblasts have altered expression of mechanosensitive genes in response to mechanical stress $(43,44)$. $L M N A$ mutations variably affect skeletal muscle, and skeletal muscle from both $L M N A$ mouse models and human patients with LMNA mutations reveal aberrant expression of electrical activity-dependent genes and epigenetic chromatin modifications, indicating that components of the LINC complex are crucial for an appropriate transcriptional response to mechanical stress (45).

To further understand how the LINC complex affects gene expression, recent studies have examined the role the nuclear lamina plays in chromatin organization. The organization of chromatin in the nucleus is not random and is thought to play a role in transcriptional regulation. Whole chromosomes or portions of chromosomes reorganize in the nucleus, coincident with changes in gene expression. The nuclear lamina participates in the scaffolding of the chromatin through interactions between chromatin and lamins A and C. Fibroblasts from patients with $L M N A$-induced cardiomyopathy have changes in the threedimensional location of chromatin compared with normal dermal fibroblasts. This chromatin mislocalization occurs concomitant with changes in gene expression, suggesting that the spatial organization of chromatin may be important for disease pathogenesis $(46,47)$. Together, these data indicate that a functional LINC complex is critical for the heart to properly respond to constant mechanical stress, potentially by regulating gene expression or other nuclear functions.

\section{Genetic assessment and modifiers for cardiomyopathy}

As with other genetic disorders, the study of DCM is undergoing a revolution, with implementation of newer, massively parallel next-generation sequencing. Herman et al. developed an exoncapture approach to sequence the more than 350 exons that comprise TTN (8). Whole exome and whole genome approaches have now become feasible and are in the clinical domain (48). While it may not be necessary to determine the sequence of all exons or the whole genome to arrive at a genetic diagnosis, it is rapidly becoming cost effective to determine the whole exome or whole genome and then selectively investigate sequence variation in specific gene(s). This analysis expands on the current strategy, which simultaneously evaluates the sequence of many DCM genes. This expansive screening is often undertaken in a single affected family member. With identification, the index mutation is then evaluated in other family members. With expansive testing, more than one pathogenic variant or variants of unknown significance may be uncovered. Determination of pathogenicity has typically relied on segregation in family members and experimental techniques performed in research laboratories. Not all families are large enough for segregation analysis. Laboratory-based experiments, while experimentally satisfying, are not performed with any type of regulatory oversight. More importantly, with the number of 
variants currently being identified through expansive testing, such experimental validation is not possible. Future approaches to validate the pathogenicity of mutations will likely rely more heavily on computational prediction algorithms coupled tightly to better estimates of population frequency (49).

The same genetic mutation, even within families, can manifest with distinct cardiac morphology (HCM or DCM), varying age of onset, and range of arrhythmia risk. Modifiers for cardiomyopathy can include both environmental and genetic influences. Animal models are particularly useful for identifying genetic modifiers because of the availability of inbred strains, more uniform housing conditions, and the ability to make an unbiased genomewide search. Le Corvoisier and colleagues mapped modifiers of heart failure using a calsequestrin-expressing transgenic model of cardiomyopathy (50). With this approach, seven different loci were identified and one was identified as TNNI3K, a gene encoding cardiac troponin I interacting kinase (51). This gene was implicated in several traits linked to cardiac function, such as fractional shortening and ventricular diameter. These data fit well with the observation that TNNI3K is a gene expressed in cardiomyocytes.

Human studies for heart failure modifiers have utilized candidate gene and whole genome approaches. The $\beta_{1^{-}}, \beta_{2^{-}}$, and the $\alpha_{2 C^{-}}$ adrenergic receptors have common DNA variants that are associated with outcome in heart failure, including survival and exercise capacity (52-55). A genomewide association study identified a region that included the $H S P B 7$ gene and the CLCNKA gene (56, 57). Further evaluation revealed G83R, a specific SNP with functional consequences in CLCNKA (rs10927887), which was overrepresented in three cohorts of patients with heart failure, including both nonischemic and ischemic heart failure (58).

Fibrosis is an important biological modifier for cardiomyopathy and cardiopulmonary function (Figure 4). A recent study showed fibrosis in young individuals who had HCM mutations but had not yet developed hypertrophy (59). This indicates that fibrosis is an early step in HCM pathology. The TGF- $\beta$ pathway mediates fibrosis in many different disease states including the heart (60). LTBP4 was identified as a modifier using a genomewide scan in a mouse model of cardiomyopathy and muscular dystrophy (61), and additional loci were identified that differentially modified heart and muscle disease (62). Additional modifiers for cardiomyopathy and heart failure will emerge from genomewide surveys currently ongoing in many laboratories. Hopefully these modifiers will not only yield diagnostic information that may predict rate of progression and arrhythmia risk, but also indicate pathways to therapeutic intervention.

\section{Conclusions}

The genetic complexity of cardiomyopathy, especially DCM, is being increasingly understood with the application of massively parallel sequencing technologies. Genetic diagnosis is now readily accessible for DCM and provides useful information about prognosis and associated risks. The pathways uncovered from studies on genetic cardiomyopathy highlight the importance of the sarcomere, including the $\mathrm{Z}$ band, the connections to the plasma membrane, and the nuclear membrane. Modifier genes for DCM include secondary rare variants, common variants in essential pathways for heart function, and each of these affords opportunities for diagnosis and prognosis as well as therapeutic intervention.

\section{Acknowledgments}

This work was supported by the Sarnoff Foundation (to J.R. Golbus) and NIH grants HL61322, HL092443, AR052646, and NS072027 (to E.M. McNally and M.J. Puckelwartz).

Address correspondence to: Elizabeth M. McNally, University of Chicago, 5841 S. Maryland MC6088, Chicago, Illinois 60637, USA. Phone: 773.702.2672; Fax: 773.702.2681; E-mail: emcnally@ uchicago.edu.
1. Codd MB, Sugrue DD, Gersh BJ, Melton LJ 3rd. Epidemiology of idiopathic dilated and hypertrophic cardiomyopathy. A population-based study in Olmsted County, Minnesota, 1975-1984. Circulation. 1989;80(3):564-572.

2. Miura K, et al. Epidemiology of idiopathic cardiomyopathy in Japan: results from a nationwide survey. Heart. 2002;87(2):126-130.

3. Maron BJ. Hypertrophic cardiomyopathy: a systematic review. JAMA. 2002;287(10):1308-1320.

4. Wilkinson JD, et al. The pediatric cardiomyopathy registry and heart failure: key results from the first 15 years. Heart Fail Clin. 2010;6(4):401-413, vii.

5. Petretta M, Pirozzi F, Sasso L, Paglia A, Bonaduce D. Review and metaanalysis of the frequency of familial dilated cardiomyopathy. Am J Cardiol. 2011;108(8):1171-1176.

6. Ingles J, Doolan A, Chiu C, Seidman J, Seidman C, Semsarian C. Compound and double mutations in patients with hypertrophic cardiomyopathy: implications for genetic testing and counselling. J Med Genet. 2005;42(10):e59.

7. Girolami F, et al. Clinical features and outcome of hypertrophic cardiomyopathy associated with triple sarcomere protein gene mutations. J Am Coll Cardiol. 2010;55(14):1444-1453.

8. Herman DS, et al. Truncations of titin causing dilated cardiomyopathy. $N$ Engl J Med. 2012;366(7):619-628

9. Fiset C, Giles WR. Cardiac troponin T mutations promote life-threatening arrhythmias. J Clin Invest. 2008;118(12):3845-3847.

10. Kamisago M, et al. Mutations in sarcomere protein genes as a cause of dilated cardiomyopathy. $N$ Engl JMed. 2000;343(23):1688-1696.

11. Hanson EL, et al. Cardiac troponin T lysine 210 deletion in a family with dilated cardiomyopathy. J Card Fail. 2002;8(1):28-32.

12. Du CK, et al. Knock-in mouse model of dilated cardiomyopathy caused by troponin mutation. Circ Res. 2007;101(2):185-194.

13. Dyer EC, et al. Functional analysis of a unique troponin c mutation, GLY159ASP, that causes familial dilated cardiomyopathy, studied in explanted heart muscle. Circ Heart Fail. 2009;2(5):456-464.

14. Lakdawala NK, et al. Familial dilated cardiomyopathy caused by an alpha-tropomyosin mutation: the distinctive natural history of sarcomeric dilated cardiomyopathy. J Am Coll Cardiol. 2010;55(4):320-329.

15. Tardiff JC. Thin filament mutations: developing an integrative approach to a complex disorder. Circ Res. 2011;108(6):765-782.

16. Schmitt JP, et al. Cardiac myosin missense mutations cause dilated cardiomyopathy in mouse models and depress molecular motor function. Proc Natl Acad Sci U S A. 2006;103(39):14525-14530.

17. Klaassen $S$, et al. Mutations in sarcomere protein genes in left ventricular noncompaction. Circulation. 2008;117(22):2893-2901.

18. Dellefave LM, et al. Sarcomere mutations in cardiomyopathy with left ventricular hypertrabeculation. Circ Cardiovasc Genet. 2009;2(5):442-449.

19. LeWinter MM, Wu Y, Labeit S, Granzier H. Cardiac titin: structure, functions and role in disease. Clin Chim Acta. 2007;375(1-2):1-9.
20. Gautel M. Cytoskeletal protein kinases: titin and its relations in mechanosensing. Pflugers Arch. 2011;462(1):119-134.

21. Gerull B, et al. Mutations of TTN, encoding the giant muscle filament titin, cause familial dilated cardiomyopathy. Nat Genet. 2002;30(2):201-204.

22. Xu X, et al. Cardiomyopathy in zebrafish due to mutation in an alternatively spliced exon of titin. Nat Genet. 2002;30(2):205-209.

23. Miller MK, Granzier H, Ehler E, Gregorio CC. The sensitive giant: the role of titin-based stretch sensing complexes in the heart. Trends Cell Biol. 2004;14(3):119-126.

24. Nagueh SF, et al. Altered titin expression, myocardial stiffness, and left ventricular function in patients with dilated cardiomyopathy. Circulation. 2004;110(2):155-162.

25. Taylor $M$, et al. Genetic variation in titin in arrhythmogenic right ventricular cardiomyopathy-overlap syndromes. Circulation. 2011;124(8):876-885.

26. Hayashi $\mathrm{T}$, et al. Tcap gene mutations in hypertrophic cardiomyopathy and dilated cardiomyopathy. J Am Coll Cardiol. 2004;44(11):2192-2201.

27. Mohapatra B, et al. Mutations in the muscle LIM protein and alpha-actinin-2 genes in dilated cardiomyopathy and endocardial fibroelastosis. Mol Genet Metab. 2003;80(1-2):207-215.

28. Knoll R, et al. The cardiac mechanical stretch sensor machinery involves a $\mathrm{Z}$ disc complex that is defective in a subset of human dilated cardiomyopathy. Cell. 2002;111(7):943-955.

29. Moulik M, et al. ANKRD1, the gene encoding cardiac ankyrin repeat protein, is a novel 
dilated cardiomyopathy gene. J Am Coll Cardiol. 2009;54(4):325-333

30. Duboscq-Bidot L, et al. Mutations in the ANKRD1 gene encoding CARP are responsible for human dilated cardiomyopathy. Eur Heart J. 2009;30(17):2128-2136.

31. Purevjav E, et al. Molecular basis for clinical heterogeneity in inherited cardiomyopathies due to myopalladin mutations. Hum Mol Genet 2012;21(9):2039-2053.

32. Hassel D, et al. Nexilin mutations destabilize cardiac Z-disks and lead to dilated cardiomyopathy. Nat Med. 2009;15(11):1281-1288.

33. Lapidos KA, Kakkar R, McNally EM. The dystrophin glycoprotein complex: signaling strength and integrity for the sarcolemma. Circ Res. 2004;94(8):1023-1031.

34. Yasuda S, Townsend D, Michele DE, Favre EG, Day SM, Metzger JM. Dystrophic heart failure blocked by membrane sealant poloxamer. Nature. 2005;436(7053):1025-1029.

35. Duboc D, Meune C, Lerebours G, Devaux JY, Vaksmann G, Becane HM. Effect of perindopril on the onset and progression of left ventricular dysfunction in Duchenne muscular dystrophy. J Am Coll Cardiol. 2005;45(6):855-857.

36. Duboc D, et al. Perindopril preventive treatment on mortality in Duchenne muscular dystrophy: 10 years' follow-up. Am Heart J. 2007;154(3):596-602.

37. Malhotra R, Mason PK. Lamin A/C deficiency as a cause of familial dilated cardiomyopathy. Curr Opin Cardiol. 2009;24(3):203-208.

38. Arbustini E, et al. Autosomal dominant dilated cardiomyopathy with atrioventricular block: a lamin A/C defect-related disease. J Am Coll Cardiol. 2002;39(6):981-990.

39. van Rijsingen IA, et al. Risk factors for malignant ventricular arrhythmias in lamin a/c mutation carriers a European cohort study. J Am Coll Cardiol. 2012;59(5):493-500.

40. Mellad JA, Warren DT, Shanahan CM. Nesprins
LINC the nucleus and cytoskeleton. Curr Opin Cell Biol. 2011;23(1):47-54.

41. Puckelwartz MJ, et al. Nesprin-1 mutations in human and murine cardiomyopathy. J Mol Cell Cardiol. 2010;48(4):600-608.

42. Zhang Q, et al. Nesprin-1 and -2 are involved in the pathogenesis of Emery Dreifuss muscular dystrophy and are critical for nuclear envelope integrity. Hum Mol Genet. 2007;16(23):2816-2833.

43. Lammerding J, Hsiao J, Schulze PC, Kozlov S, Stewart CL, Lee RT. Abnormal nuclear shape and impaired mechanotransduction in emerin-deficient cells. J Cell Biol. 2005;170(5):781-791.

44. Lammerding J, et al. Lamin A/C deficiency causes defective nuclear mechanics and mechanotransduction. J Clin Invest. 2004;113(3):370-378.

45. Mejat A, et al. Lamin A/C-mediated neuromuscular junction defects in Emery-Dreifuss muscular dystrophy. J Cell Biol. 2009;184(1):31-44

46. Meaburn KJ, et al. Primary laminopathy fibroblasts display altered genome organization and apoptosis. Aging Cell. 2007;6(2):139-153.

47. Mewborn SK, et al. Altered chromosomal positioning, compaction, and gene expression with a lamin A/C gene mutation. PloS one. 2010;5(12):e14342.

48. Gahl WA, et al. The National Institutes of Health Undiagnosed Diseases Program: insights into rare diseases. Genet Med. 2012;14(1):51-59.

49. Norton N, et al. Evaluating pathogenicity of rare variants from dilated cardiomyopathy in the exome Era. Circ Cardiovasc Genet. 2012;5(2):167-174.

50. Le Corvoisier P, Park HY, Carlson KM, Marchuk DA, Rockman HA. Multiple quantitative trait loci modify the heart failure phenotype in murine cardiomyopathy. Hum Mol Genet. 2003; 12(23):3097-3107.

51. Wheeler FC, et al. Tnni3k modifies disease progression in murine models of cardiomyopathy. PLoS Genet. 2009;5(9):e1000647.

52. Liggett SB, et al. The Ile164 beta2-adrenergic receptor polymorphism adversely affects the outcome of congestive heart failure. J Clin Invest. 1998;102(8):1534-1539.

53. Wagoner LE, et al. Polymorphisms of the beta(2)-adrenergic receptor determine exercise capacity in patients with heart failure. Circ Res. 2000;86(8):834-840.

54. Small KM, Wagoner LE, Levin AM, Kardia SL, Liggett SB. Synergistic polymorphisms of beta1- and alpha2C-adrenergic receptors and the risk of congestive heart failure. $N$ Engl J Med. 2002;347(15):1135-1142.

55. Mialet Perez J, et al. Beta 1-adrenergic receptor polymorphisms confer differential function and predisposition to heart failure. Nat Med. 2003;9(10):1300-1305.

56. Cappola TP, et al. Common variants in HSPB7 and FRMD4B associated with advanced heart failure. Circ Cardiovasc Genet. 2010;3(2):147-154.

57. Villard E, et al. A genome-wide association study identifies two loci associated with heart failure due to dilated cardiomyopathy. Eur Heart J. 2011;32(9):1065-1076.

58. Cappola TP, et al. Loss-of-function DNA sequence variant in the CLCNKA chloride channel implicates the cardio-renal axis in interindividual heart failure risk variation. Proc Natl Acad Sci U S A. 2011;108(6):2456-2461.

59. Ho CY, et al. Myocardial fibrosis as an early manifestation of hypertrophic cardiomyopathy. $\mathrm{NEnglJ}$ Med. 2010;363(6):552-563.

60. Dobaczewski M, Chen W, Frangogiannis NG. Transforming growth factor (TGF)-beta signaling in cardiac remodeling. $J \mathrm{Mol}$ Cell Cardiol. 2011;51(4):600-606

61. Heydemann A, et al. Latent TGF-beta-binding protein 4 modifies muscular dystrophy in mice. J Clin Invest. 2009;119(12):3703-3712.

62. Swaggart KA, Heydemann A, Palmer AA, McNally EM. Distinct genetic regions modify specific muscle groups in muscular dystrophy. Physiol Genomics. 2011;43(1):24-31. 